\title{
Molecular cloning and sequencing of rat Cdc42 GTPase cDNA
}

\author{
Joong-Soo Han ${ }^{1,5}$, Jong-Hoon Kim ${ }^{1}$, \\ Jong Gon Kim¹, Jae-Bong Park², \\ Dong-Young $\mathrm{Noh}^{3}$ and Kweon-Haeng Lee ${ }^{4}$ \\ ${ }^{1}$ Institute of Biomedical Science and Department of Biochemistry, \\ College of Medicine, Hanyang University, Seoul, Korea \\ ${ }^{2}$ Department of Biochemistry, College of Medicine, \\ Hanlym University, Chuncheon, Kangwondo, Korea \\ ${ }^{3}$ Department of Surgery, College of Medicine, \\ Seoul National University, Seoul, Korea \\ ${ }^{4}$ Department of Pharmacology, College of Medicine, \\ The Catholic University of Korea, Seoul, Korea \\ ${ }^{5}$ Corresponding author: Tel, +82-2-2290-0623; \\ Fax, +82-2-2294-6270; E-mail, jshan@email.hanyang.ac.kr
}

Accepted 22 August 2000

\begin{abstract}
Cdc42 is a member of the Rho family of small GTPase and plays an important role in intracellular signaling pathways regulating cell morphology, motility and stimulation of DNA synthesis. We have isolated cDNA encoding Cdc42 from a rat brain cDNA library using PCR-cloning strategy. The sequence of isolated gene revealed an open reading frame of 576 nucleotides with the potential to encode a protein of 191 amino acids with a predicted molecular weight of $21 \mathrm{kD}$. The resulting sequence was incorporated into the GenBank with accession number, AF205635. Sequence analysis revealed that overall cDNA sequence identity is $96 \%$ with human G25K and $52 \%$ with rat Chp, a homologue of the GTPase human Cdc42Hs, and having one nucleotide difference from the mouse Cdc42. However, putative protein sequence was identical to the mouse and human brain $\mathrm{Cdc42Hs}$. On expression of the cDNA in COS-7 cells, a protein molecular weight of $21 \mathrm{kD}$ was detected in immunoblotting using anti-human Cdc42 antibodies. Therefore, these results suggest that the cDNA we are reporting is most likely the rat homologue of the GTPase human Cdc42.
\end{abstract}

Keywords: GTPase, Cdc42, PCR Cloning, COS-7 cell, Rho

\section{Introduction}

Small GTPases are essential components in a number of biological processes, including the hormonal regula- tion of adenylate cyclase and phospholipase, visual transduction, protein trafficking, secretion and cell growth (Shinjo et al., 1990; Johnson, 1999). Cdc42 is a member of the Rho family of small GTPase and plays an important role in intracellular signaling pathways that influence cell morphology, motility and stimulation of DNA synthesis (Nobes and Hall, 1995; Olson et al., 1995). Like other GTPases, Cdc42 can exist in an active GTPbound state and an inactive GDP-bound state (Bourne et al., 1990). In yeast, Cdc42 acts as a regulator of the cell division cycle (Adams et al., 1990; Oehlen and Cross, 1998). The mammalian homologue of yeast Cdc42 has been shown to regulate the formation of the actin structure filopodia in Swiss 3T3 cells (Kozma et al., 1995) and cell polarity-dependent processes in T cell activation (Stowers et al., 1995). Human Cdc42 was initially isolated in platelets and placenta, and serves as a substrate for phosphorylation by epidermal growth factor receptor (Evans et al., 1986). In addition, it has recently been shown that Cdc42 may act downstream of Arf in some types of intracellular trafficking events (Radhakrishna et al., 1999) and can act synergistically with Arf to stimulate phospholipase $D$ activity in mammalian cells (Han et al., 1996). Structural and functional Cdc42 homologues have subsequently been characterized in the pathogenic yeast Candida albicans (Mirbod et al., 1997), S. pombe (Miller and Johnson, 1994), C. elegans (Chen et al., 1993), Drosophila (Luo et al., 1994), chicken (Gallus gallus) cochlea (Gong et al., 1997), mouse (Mus musculus) liver (Gong et al., 1997) and brain (Marks and Kwiatkowski, 1996). These homologues are 80 to $95 \%$ identical in predicted amino acid sequence (Johnson, 1999) and it suggests that Cdc42 may have conserved functions in various species. In the rat, although the Chp, a homologue of the GTPase human $\mathrm{Cdc} 42 \mathrm{Hs}$, was cloned, its overall sequence identity is $52 \%$ with Cdc42Hs (Aronheim et al., 1998). We have recently purified $\mathrm{Cdc} 42$ from rat brain cytosol (Han et al., 1998). Here we report the cloning and sequencing of cDNA which encodes the rat (Rattus norverticus) Cdc42 from a rat brain cDNA library. Using the PCRcloning technique, we successfully cloned cDNA for rat Cdc42. The cDNA obtained could be expressed in COS-7 cells, and Western blot analysis showed that the protein was highly reactive to anti-human Cdc42 antibodies.

\section{Materials and Methods}

PCR cloning of the rat Cdc42 cDNA

$A$ rat brain cDNA library in $P B 42 A D$ was used as the 
template for PCR-cloning of rat Cdc42 cDNA. Oligonucleotide primers were arranged as follows based on the mouse Cdc42 cDNA: 5'-CCGGGATCCATGCAGACAATTAAGTGTGTTGTT-3' (sense) and 5'-GCCGAATTCTTAGAATATACAGCACTTCCTTTT-3' (antisense) corresponding to nucleotides 1-576 of the full length of mouse Cdc42. The sequences on underlines are BamH I (GGATCC) and ECoR I (GAATTC) recognition sites which were added to the insert PCR products into expression vector, pcDNA3.1 (Invitrogen). The thermal cycler program used for PCR amplification consisted of an initial denaturation step at $94^{\circ} \mathrm{C}$ followed by 30 cycles consisting of 1 min denaturation at $94^{\circ} \mathrm{C}, 1$ min annealing at $54^{\circ} \mathrm{C}$ and a $45 \mathrm{sec}$ elongation at $72^{\circ} \mathrm{C}$. A final $10 \mathrm{~min}$ elongation at $72^{\circ} \mathrm{C}$ followed. The PCR products were separated on $1 \%$ agarose gels and recovered from gels using JETsorb (Genomed). For the sequence analysis, the PCR product was cloned into TA-pGEM cloning vector (Promega), and E. coli bacterial strain JM109 was used as a host.

\section{DNA sequencing}

Plasmid DNA was isolated from recombinant colonies with the plasmid mini-prep kit (Promega). The plasmid DNA was purified and sequenced by dideoxy chain termination method using T7/SP6 sequencing primers and BigDye Terminator Cycle Sequencing Kit with $A B I$ PRISM 310 Genetic Analyzer (PE Applied Biosystems). The sequences were subsequently analyzed with the program DNASIS version 2.1 (Hitachi Software).

\section{Cell culture}

COS-7 cell was obtained from American Type Culture Collection (ATCC) and grown in DMEM (Gibco) supplement with $10 \%$ heat-inactivated fetal bovine serum, penicillin $(100 \mathrm{unit} / \mathrm{ml})$ and streptomycin $(100 \mu \mathrm{g} / \mathrm{ml})$ at $37^{\circ} \mathrm{C}$ in $\mathrm{CO}_{2}$ incubators.

\section{Transient transfection of COS-7 Cells using Lipofec- AMINE}

The cDNA fragments encoding Cdc42 were subcloned in the mammalian expression vector, pcDNA3.1. The TA-pGEM cloning vector containing Cdc42 cDNA were double-digested with BamH I and Not I, and the resulting CDNA were ligated into the corresponding sites in pcDNA3.1 using $T_{4}$ DNA ligase. COS-7 cells were transfected with the recombinant plasmid pcDNA3.1-Cdc42 using LipofectAMINE reagent (Gibco BRL) according to the manufacturer's protocol. Briefly, cells were grown to $80 \%$ confluence in $100-\mathrm{mm}$ dishes. cDNA $(2 \mu \mathrm{g})$ and LipofecAMINE $(20 \mu \mathrm{l})$ were separately diluted in serumfree DMEM to $200 \mu$ volumes, mixed, and incubated at room temperature for $1 \mathrm{~h}$. Cells were washed twice with PBS and overlaid with $5 \mathrm{ml}$ of serum-free DMEM to which the DNA-lipid complexes were added. After $6 \mathrm{~h}$, the DNA-lipid complex was replaced with a growth medium containing $10 \%$ FBS and $500 \mu \mathrm{g} / \mathrm{ml}$ geneticin, and the cells were incubated for $72 \mathrm{~h}$.

\section{Immunoblot analysis}

Cells were washed twice with phosphate-buffered saline

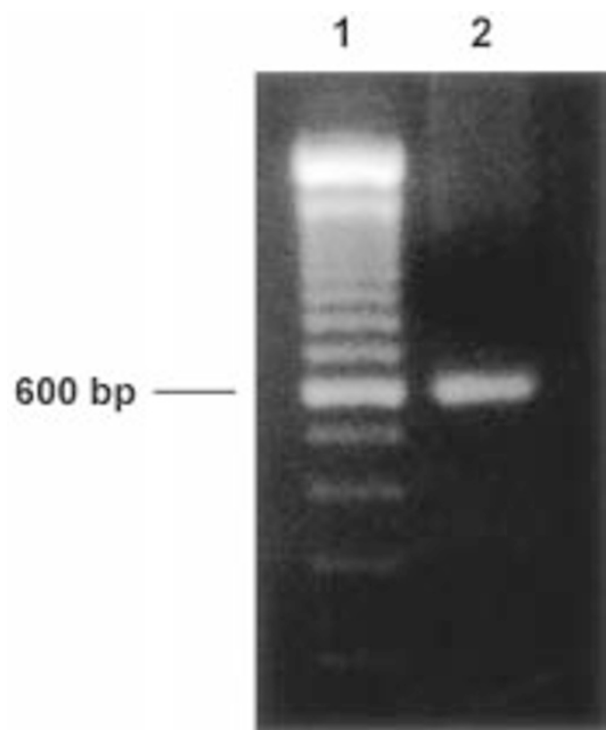

Figure 1. Analysis of amplified PCR products by $1.5 \%$ agarose gel electrophoresis. Lane 1: 100 bp size marker, Lane 2: PCR product from rat brain library obtained after 30 cycles at $94^{\circ} \mathrm{C} 1 \mathrm{~min}, 54^{\circ} \mathrm{C} 1 \mathrm{~min}, 72^{\circ} \mathrm{C} 45$ sec.

1 ATGCAGACAATTAAGTGTGTTGTTGTTGGTGATGGTGCTGTTGGTAAAACATGTCTCCTG

$\begin{array}{llllllllllllllllllllll}1 & M & Q & T & I & K & C & V & V & V & G & D & G & A & V & G & K & T & C & L & L\end{array}$

61 ATATCCTACACAACAAACAAATTCCCATCGGAATATGTACCAACTGTTTTTGACAACTAT

$\begin{array}{lllllllllllllllllllll}21 & I & S & Y & T & T & N & K & F & P & S & E & Y & V & P & T & V & F & D & N & Y\end{array}$

121 GCAGTCACAGTTATGATTGGTGGAGAGCCATACACTCTTGGACTTTTTGATACTGCAGGG

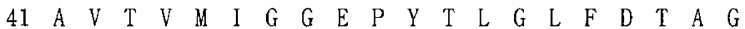

181 CAAGAGGATTATGACAGACTACGACCGCTAAGTTATCCACAGACAGATGTTTTTCTAGTA

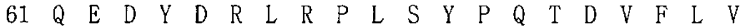

241 TGTTTCTCAGTGGTCTCTCCATCCTCATTTGAAAATGTGAAAGAAAAGTGGGTGCCTGAG $\begin{array}{llllllllllllllllllllll}81 & C & F & S & V & V & S & P & S & S & F & E & N & V & K & E & K & W & V & P & E\end{array}$

301 ATAACTCACCACTGTCCAAAGACTCCTTTCTTGCTTGTTGGGACCCAAATTGATCTCAGA

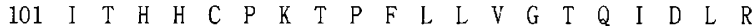

361 GATGACCCCTCTACTATTGAGAAACTTGCCAAGAACAAACAGAAGCCTATTACTCCAGAG $\begin{array}{lllllllllllllllllllll}121 & D & D & P & S & T & I & E & K & L & A & K & N & K & Q & K & P & I & T & P & E\end{array}$

421 ACTGCTGAAAAGCTGGCGCGGGATCTGAAGGCTGTCAAGTATGTGGAGTGCTCCGCCCTC

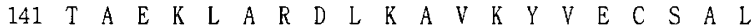

481 ACACAGAGAGGTCTGAAGAATGTGTTTGATGAGGCTATCCTAGCTGCCCTCGAGCCTCCG $\begin{array}{lllllllllllllllllllll}161 & T & Q & R & G & L & K & N & V & F & D & E & A & I & L & A & A & L & E & P & P\end{array}$

541 GAAACTCAACCCAAAAGGAAGTGCTGTATATTCTAA

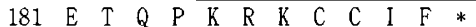

Figure 2. Nucleotide and deduced amino acid sequences of the rat brain Cdc42. Nucleotide and amino acid positions are indicated at the left and the termination codon is marked by an asterisk. The primers used for PCR span the entire coding region of the cDNA and are underlined. The accession number of this CDNA in the GenBank is AF205635. 


\begin{tabular}{|c|c|c|}
\hline & $\ldots \ldots \ldots$ & \\
\hline $\mathrm{Cdc} 42 \mathrm{mb}$ & $\ldots$ & \\
\hline G25K & A I G & \\
\hline & G G G A G C T G A G C G A G & \\
\hline$=42 \mathrm{rb}$ & $\ldots$ A T TAAGT & \\
\hline c. $42 \mathrm{mb}$ & ( & 16 \\
\hline G25K & - & \\
\hline$n p$ & 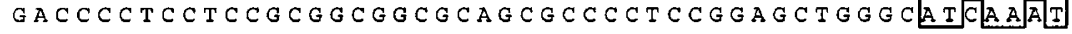 & \\
\hline $\mathrm{c} 42 \mathrm{rb}$ & GTGTTGTTGTTGGTGATGGTGCTGTTGGTAAAACATG TCTCTGATATC & \\
\hline cdc4 $2 \mathrm{mb}$ & G T IT T T T T T TGTGATGGTGCTGTIGGTAAAACATGTCTCCTGATATCC & \\
\hline $5 \mathrm{~K}$ & 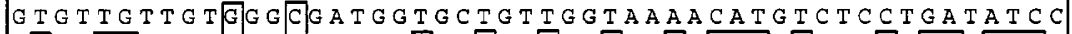 & \\
\hline Chp & 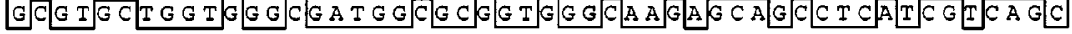 & \\
\hline $\mathrm{Cdc} 42 \mathrm{rb}$ & TACACAACAAACAAATTCCCATCGGAATATGTACCAACTGTTTTTGACAA & \\
\hline $\mathrm{Cdc} 42 \mathrm{mb}$ & TACACAACAAACAAATTCCCATCGGAATATGTACCAA & 116 \\
\hline G25K & TACACAACAAACAAATTTC CATCGGAATATGTACCG & 116 \\
\hline Chp & 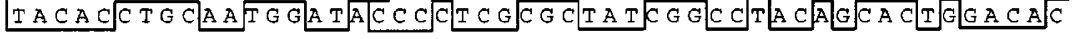 & \\
\hline $\mathrm{dc} 42 \mathrm{rb}$ & C TATGCAGTCACAGTTATGATTGGTGGAGAG & \\
\hline Cdc $42 \mathrm{mb}$ & CTATGCAGTCACAGTTATGATTGGTGGAGAG & 165 \\
\hline G25K & CTATGCAGTCACAGTTATGATTGGTGGAGA & 165 \\
\hline Chp & 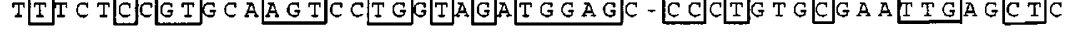 & \\
\hline $\mathrm{cdc} 42 \mathrm{rb}$ & T T T G A T A C T G CA G G G CA A G & \\
\hline $\mathrm{cdc} 42 \mathrm{mb}$ & T T T GATACT GCAGGGCAAGAGGAT TATGACA GACTA & 15 \\
\hline $\mathrm{G} 25 \mathrm{~K}$ & 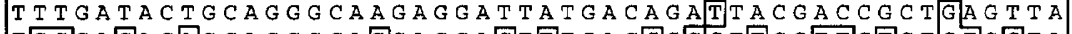 & 215 \\
\hline Chp & 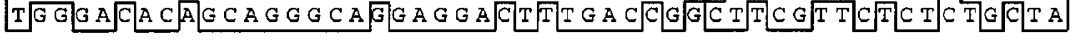 & \\
\hline 2de42 2 & T C CACA & \\
\hline $\mathrm{Cdc} 42 \mathrm{mb}$ & TCCACAGACAGATGTTTTTCTAGTATGTTTCTCAGTGGTCTCTCCATCCT & 265 \\
\hline $\mathrm{G} 25 \mathrm{~K}$ & 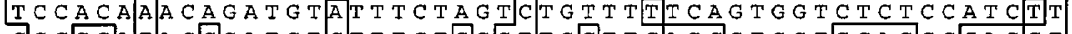 & 265 \\
\hline ap & C CGA T G T CT T T C T GG T T GCT T C A G C T G G T G C A GC C C A G C T & \\
\hline & & \\
\hline Cac42 & C C AC I & 315 \\
\hline $525 \mathrm{~K}$ & C C T G A G A TAACT CA C CACT T T & 315 \\
\hline & C C]G G A G A T C C G C A C T[C A C A A C & \\
\hline
\end{tabular}

CdC42rb C CA A A ACT CCT T TCT T C C T T G T T GGGACCCAAATT GATCTCAGAGATGA 365 Cdc4 $2 \mathrm{mb}$ C CAAAGACTCCTTTCTTGCTTGTTGGGACC CAAATTGATCTCAGAGATGA 365 G25K C CAAAGACTCCTTTCTTGCTTGTTGGGACTCAAATTGATCTCAGAGATGA 365

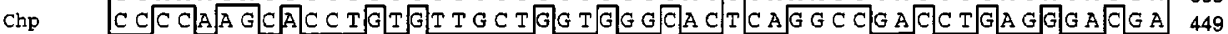

CdC42rb CCCCTCTACTATTGAGAAACTTG- CCAAGAACAAACAGAAG-CCTATTA 412

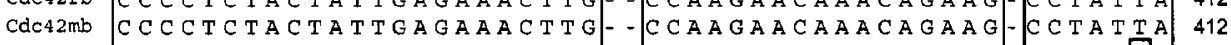
$625 \mathrm{~K}$ C C C CTCTACTATTGAGAAACTTG--C CAAGAACAAACAGAAG-C CTATCA 412

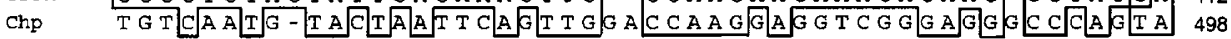

Cdc42rb CTCCAGAGACT-GCTGAAAACTGGCGCGGGATCTGAAGGTGTCAAGTA 461 Cdc42mb C T C CAGAGACT GCTGAAAACTGGCGCGGGATCTGAAGGCTGTCAAGTA 461 G25K CTCCAGAGACT-G CTGAAAAGCTGGCCCGTA CCTGAAGGTGTCAAGTA 461

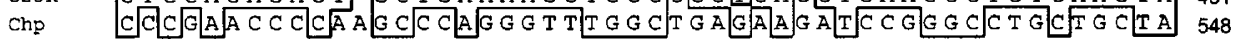

CdC42rb TGTGGAGTGCTCCGCCCTCACACAGAGAGGTCTGAAGAATGTGTTTGATG 511 Cdc42mb TGTGGAGTGCTCTCCCTCACACAGAGAGGTCTGAAAATTGTTTATG 511 G25K TGTGGAGTGTTCTCACTTACACAGAGAGGTCTGAAGAATGTGTTTGATG 511

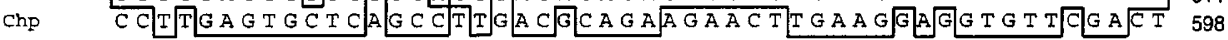

Cdc42rb AGGCTATCCTAGCTGCCCTCGAGCCT . . . CCG . . . . . . GAAACTC 547

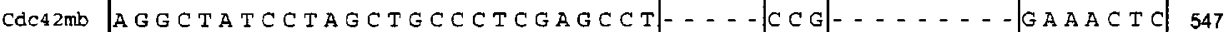
G25K A GGCTATC CTAGCTGCCCTCGAGCCT - . . C C G

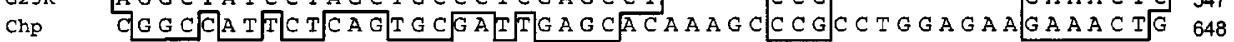

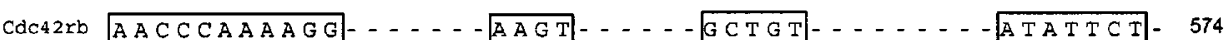
Cdc42mb A A C C CAAAAG G25K A A C C CAAAAGG

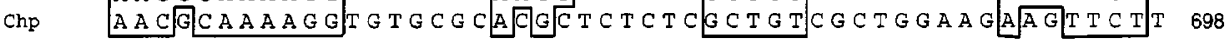

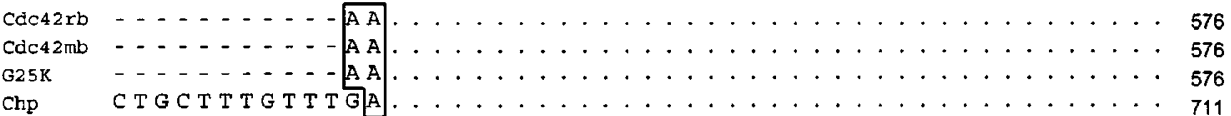

Figure 3. Multiple sequence alignment of $\mathrm{Cdc} 42$ or its homologues. The nucleotide sequences of rat brain $\mathrm{Cdc} 42$ (Cdc42rb), mouse brain $\mathrm{Cdc42}$ (Cdc42mb), human G25K (G25K, from human fetal brain) and rat Chp (Chp), a homologue of the GTPase human Cdc42Hs were aligned using the program DNASIS Version 2.1. The boxed region denotes the nucleotides identical to those of the rat Cdc42 cDNA and dashes indicate gaps introduced into the sequence to improve alignment. 
(PBS) and harvested in $0.5 \mathrm{ml}$ of lysis buffer $(50 \mathrm{mM}$ $\mathrm{NaCl}, 1 \mathrm{mM} \mathrm{MgCl}$, 2 mM EDTA, $1 \mathrm{mM}$ dithiothreitol, 1 $\mathrm{mM}$ phenylmethylsulfonyl fluoride, $10 \mu \mathrm{g} / \mathrm{ml}$ aprotinin, $10 \mu \mathrm{g} / \mathrm{ml}$ leupeptin, $50 \mathrm{mM}$ HEPES, $\mathrm{pH}$ 7.5) and disrupted by sonication. Total protein concentration was determined by the Bradford procedure using BSA as a standard. Sample lysate $(40 \mu \mathrm{g})$ was mixed with sample buffer (4\% SDS, $10 \% \beta$-mercaptoethanol, $20 \%$ glycerol in $0.125 \mathrm{M}$ Tris, $\mathrm{pH}$ 6.8) containing bromophenol blue, boiled for $5 \mathrm{~min}$, and then run on $12 \%$ SDS-PAGE. The separated protein was electrophoretically transferred to the nitrocellulose membrane (BA83, Schleicher \& Schuell). The membrane was then blocked with 5\% BSA in TTBS (Tris-buffered saline containing $0.01 \%$ Tween-20) for $1 \mathrm{~h}$ and then incubated for another $1 \mathrm{~h}$ with mouse antihuman Cdc42 monoclonal antibody at $1: 1000$ dilution. Unbound primary antibodies were removed by three washings with TTBS, and the membrane was incubated with peroxidase-conjugated anti-mouse IgG antibody (Sigma) for $1 \mathrm{~h}$. After washing, the membrane was developed by enhanced chemiluminesence (Amersham Corp.) and exposed for 1 min to Kodak X-AR5 film prior to developing the immunoblots.

\section{Results and Discussion}

In this report, we have successfully isolated and sequenced the cDNA for a low molecular weight GTPbinding protein, $\mathrm{Cdc} 42$ from a rat brain library. Recently, a homologue of the GTPase human $\mathrm{Cdc} 42 \mathrm{Hs}$, designated Chp, was cloned from a rat pituitary cDNA library (Aronheim et al., 1998). However, Chp protein exhibits an overall sequence identity of $52-57 \%$ to Cdc42 of other species. To clone the Cdc42 in the rat, we screened an adult rat brain CDNA library using the PCRcloning technique. Recent studies showed that the TA cloning system is the most efficient cloning system for PCR-amplified DNA fragments with minimal manipulation and ease of handling. Simultaneously, the cloned DNA obtained from the TA cloning system can be directly sequenced without further manipulation (Kern et al., 1992; Sarkar et al., 1993).

By using the primer based on the mouse Cdc42 CDNA, we obtained a PCR product which was about 600 bp (Figure 1). The resulting PCR product was ligated into a TA-pGEM cloning vector, and the positive clones from PCR reactions were subjected to nucleotide sequencing. Sequencing of the inserted PCR product revealed that it has an open reading frame of $576 \mathrm{bp}$ that codes for 191 amino acids, yielding a predicted $M_{\mathrm{r}}$ 21,000 . The nucleotide sequence and the deduced amino acids are shown in Figure 2. Overall CDNA sequence identity is $96 \%$ with human G25K, and $52 \%$ with rat

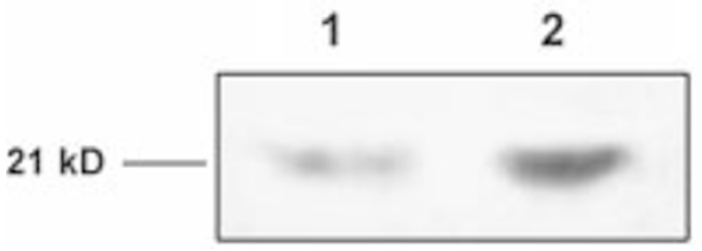

Figure 4. Reactivity of the COS-7-expressed rat Cdc42 protein with antihuman $\mathrm{Cdc} 42$ antibodies. The rat brain $\mathrm{Cdc} 42$ protein was expressed in COS-7 cells with pcDNA 3.1 vector, and cell lysates were subjected to SDSPAGE. The gel was immnoblotted with anti-human Cdc42 antibodies and the blot was developed with enhanced chemiluminescence. Lane 1: COS7 cells transfected with vector lacking the cloned CDNA inserts, Lane 2: COS-7 cell expressing cloned rat Cdc42 $(100 \mu \mathrm{g})$.

Chp, a homologue of the GTPase Cdc42Hs. Especially, comparison of the nucleotide sequences of cloned cDNA to those of mouse brain Cdc42 showed that they were identical except for just one nucleotide difference at position $474(T \rightarrow C)$ (Figure 3). However, putative protein sequence was identical to the mouse and human brain Cdc42 reported by Marks et al. and Barfod et al., respectively. (GenBank accession No. L78075; M35543). Previous studies have also shown that the Cdc42 family of proteins has 11 members ranging in size from 190 to 192 amino acids. Within this family, there is a very high degree of sequence conservation, ranging from $75 \%$ amino acid identity between C. albicans Cdc42p and the human brain isoform G25K to $100 \%$ identity between the mouse brain and human brain (G25K) isoforms (Barfod et al., 1993; Johnson, 1999).

To confirm our results, we transfected COS-7 cells with the cloned cDNA and investigated for cross-reactivity using anti-human Cdc42 antibody. Successful expression of the rat Cdc42 was achieved using mammalian COS-7 cells. As shown in Figure 4, COS-7 cells transfected with pcDNA3.1-Cdc42 highly expressed the protein, which was able to react strongly with anti-human Cdc42 antibodies. This antibody is reactive with epitope corresponding to amino acids 166-182 mapping near the carboxy terminus of human Cdc42 (identical to corresponding mouse sequence) and is non cross-reactive with Rac1, Rac2 or other Ras superfamily GTP binding proteins. Therefore, this result confirmed that the CDNA we are reporting is most likely the homologue of the GTPase human Cdc42.

\section{Acknowledgements}

This work was supported by a grant No. 96-0403-0201-3 from the Korea Science and Engineering Foundation. We thank Laboratory for Clinical Investigation, Hanyang University for providing ABI Prism 310 genetic analyzer (PE Applied Biosystems). 


\section{References}

Adams, A. E., Johnson, D. I., Longnecker, R. M., Sloat, B. F. and Pringle, J. R. (1990) CDC42 and CDC43, two additional genes involved in budding and the establishment of cell polarity in the yeast Saccharomyces cerevisiae. J. Cell. Biol. 111: $131-142$

Aronheim, A., Broder, Y. C., Cohen, A., Fritsch, A., Berisle, B. and Abo, A. (1998) Chp, a homologue of the GTPase Cdc42Hs, activates the JNK pathway and is implicated in reorganizing the actin cytoskeleton. Curr. Biol. 8: 1125-1128

Barfod, E. T., Aheng, Y., Kuang, W. J., Hart, M. J., Evans, T., Cerione, R. A. and Ashkenazi, A. (1993) Cloning and expression of a human CDC42 GTPase-activating protein reveals a functional SH3-binding domain. J. Biol. Chem. 268: 2605926062

Bourne, H. R., Sanders, D. A. and McCormick, F. (1990) The GTPase superfamily: a conserved switch for diverse cell functions. Nature 348: 125-132

Chen, W., Lim, H. H. and Lim, L. (1993) The CDC42 homologue from Caenorhabditis elegans. J. Biol. Chem. 268: 13280-13285

Evans, T., Brown, M. L., Fraser, E. D. and Northup, J. K. (1986) Purification of the major GTP-binding proteins from human placental membranes. J. Biol. Chem. 261: 7052-7059

Gong, T. W., Shin, J. J., Burmester, M. and Lomax, M. I. (1997) Complete cDNAs for CDC42 from chicken cochlea and mouse liver. Biochim. Biophy. Acta. Gene Struct. Express. 1353: 282-292.

Han, J. S., Chung, J. K., Kang, H. S., Donaldson, J., Bae, Y. S. and Rhee, S. G. (1996) Multiple forms of phospholipase D inhibitor from rat brain cytosol. J. Biol. Chem. 271: 1116311169

Han, J. S., Kim, H. C., Chung, J. K., Kang, H. S., Donaldson, J. and Koh, J. K. (1998) The potential role for Cdc42 protein from rat brain cytosol in phospholipase $\mathrm{D}$ activation. Biochem. Mol. Biol. Intl. 45: 1089-1103

Johnson, D. I. (1999) Cdc42: An essential rho-type GTPase controlling eukaryotic cell polarity. Microbiol. Mol. Biol. Rev. 63: $54-105$

Kern, M. J., Witte, D. P., Valerius, M. T., Aronow, B. J. and Potter, S. S. (1992) A novel murine homeobox gene isolated by a tissue specific PCR cloning strategy. Nucl. Acids Res. 20: 5189-5195

Kozma, R., Ahmed, S., Best, A. and Lim, L. (1995) The Ras- related protein $\mathrm{Cdc} 42 \mathrm{Hs}$ and bradykinin promote formation of peripheral actin microspikes and filopodia in Swiss 3T3 fibroblasts. Mol. Cell. Biol. 15: 1942-1952

Luo, L., Liao, Y. J., Jan, L. Y. and Jan, Y. N. (1994) Distinct morphogenetic functions of similar small GTTases: Drosophila Drac1 is involved in axonal outgrowth and myoblast fusion. Genes Dev. 8: 1787-1802

Marks, P. W. and Kwiatkowski, D. J. (1996) Genomic organization and chromosomal location of Murine Cdc42. Genomics 38: $13-18$

Miller, P. and Johnson, D. I. (1994) Cdc42p GTPase is involved in controlling polarized cell growth in Schizosaccharomyces pombe. Mol. Cell. Biol. 14: 1075-1083

Mirbod, F., Nakashima, S., Kitajima, Y., Cannon, R. D. and Nozawa, Y. (1997) Molecular cloning of a Rho family, CDC42Cagene from Candida albicans and its mRNA expression changes during morphogenesis. J. Med. Vet. Mycol. 35: 173179

Nobes, C. D. and Hall, A. (1995) Rho, rac, and cdc42 GTPases regulate the assembly of multimolecular focal complexes associated with actin stress fibers, lamellipodia, and filopodia. Cell 81: 53-62

Oehlen, L. J. W. M. and Cross, F. R. (1998) The role of cdc42 in signal transduction and mating of the budding yeast saccharomyces cerevisiae. J. Biol. Chem. 273: 8556-8559

Olson, M. F., Ashworth, A. and Hall, A. (1995) An essential role for Rho, Rac, and Cdc42 GTPases in cell cycle progression through G1. Science 269: 1270-1272

Radhakrishna, H., Al-Awar, O., Khachikian, Z. and Donaldson, J. G. (1999) ARF6 requirement for Rac ruffling suggests a role for membrane trafficking in cortical actin rearrangements. $J$. Cell Sci. 112: 855-866

Sarkar, F. H., Ball, D. E., Li, Y. W. and Crissman, J. D. (1993) Molecular cloning and sequencing of an Intron of Her-2/neu (ERBB2) gene. DNA Cell Biol. 12: 611-615

Shinjo, K., Koland, J. G., Hart, M. J., Narasimhan, V., Johnson, D. I., Evans, T. and Cerione, R. A. (1990) Molecular cloning of the gene for the human placental GTP-binding protein $G_{p}(G 25 K)$ : Identification of this GTP-binding protein as the human homologue of the yeast cell-division-cycle protein CDC42. Proc. Natl. Acac. Sci. USA 87: 9853-9857

Stowers, L., Yelon, D., Berg, L. J. and Chant, J. (1995) Regulation of the polarization of $T$ cells toward antigenpresenting cells by Ras-related GTPase CDC42. Proc. Natl. Acad. Sci. USA 92: 5027-5031 